\title{
Leukemias and Lymphomas
}

\section{Abstract 3133}

\begin{abstract}
Up to 7 Years of Follow-up of Single-Agent Ibrutinib in the Phase 1b/2 PCYC-1102 Trial of First Line and Relapsed/Refractory Patients With Chronic Lymphocytic Leukemia/ Small Lymphocytic Lymphoma

John C. Byrd, MD, Richard R. Furman, MD, Steven Coutre, $M D$, lan W. Flinn, MD, PhD, Jan A. Burger, MD, PhD, Kristie A. Blum, MD, Jeff Sharman, MD, William G. Wierda, MD, $P h D$, Weiqiang Zhao, MD, PhD, Nyla A. Heerema, PhD, Ying Luan, $P h D$, Emily A. Liu, PhD, James P. Dean, MD, PhD, and Susan M. O'Brien, $M D$
\end{abstract}

Visit http://www.bloodjournal.org/content/132/ Suppl_1/3133 for a complete list of contributor affiliations and full graphics.

Background: Ibrutinib (ibr), a first-in-class, oncedaily, covalent, Bruton's tyrosine kinase inhibitor, is approved in the US for treatment of patients (pts) with chronic lymphocytic leukemia/small lymphocytic lymphoma (CLL/SLL), including pts with del(17p). The activity and safety of ibr in this early phase pivotal study was investigated in pts receiving first line ibr and pts with relapsed/ refractory $(R / R)$ CLL/SLL. We report data on the longest follow up available, up to $7 \mathrm{y}$, for pts receiving single-agent ibr.

Methods: Phase $1 \mathrm{~b} / 2$ (PCYC-1102; NCT01105247) and the extension study (PCYC1103; NCT01109069) included pts receiving $420 \mathrm{mg}$ (first line $\mathrm{n}=26 ; \mathrm{R} / \mathrm{R} \mathrm{n}=67$ ) or $840 \mathrm{mg} \mathrm{QD}$ ibr (first line $n=5 ; R / R n=34)$ in the first line $(\geq 65 y)$ or $R / R$ ( $\geq 1$ prior therapy) settings. Ibr was administered until disease progression (PD) or unacceptable toxicity. Efficacy was evaluated by investigatorassessed overall response rate (ORR, including partial response with lymphocytosis [PR-L]) using consensus criteria for CLL and SLL, duration of response (DOR; PR-L or better), progression free survival (PFS), and overall survival (OS). Responses were assessed for Döhner hierarchical subgroups.

J Adv Pract Oncol 2019;10(suppl 2):26-32

https://doi.org/10.6004/jadpro.2019.10.2.18
Median (95\% CI) survival outcomes are reported. Adverse event (AE) monitoring was from first ibr dose until 30 days after the last dose; generally only grade $\geq 3$ AEs and other significant AEs were collected on PCYC-1103.

Results: Of 132 pts, 31 received first line ibr and 101 had R/R disease. As of the cutoff, 17 (55\%) first line and 21 (21\%) R/R pts continued ibr, with median (range) follow-up of $67 \mathrm{mo}(0.7+, 87)$. Median (range) age for all pts was 68 y (37-84); 43\% of pts were $\geq 70 \mathrm{y}$ (first line, 74\%; R/R, 34\%). Baseline genomic characteristics have been reported and included many high-risk features such as complex karyotype (first line, 13\%; R/R, 37\%) and unmutated $I G H V$ (first line, $48 \%$; R/R, 78\%). R/R pts received a median (range) of 4 prior therapies (1-12). ORR was $89 \%$ for all pts (complete response [CR], 15\%), with similar rates in first line (87\% [CR, 32\%]) and R/R pts (89\% [CR, 10\%]). Median DOR was not reached (NR; 95\% CI: not estimable [NE], NE) for first line and was $57 \mathrm{mo}$ $(38,80)$ for R/R pts. Median PFS was NR (95\% CI: NE, NE) for first line and was 51 mo $(37,70)$ for R/R pts (Fig 1); estimated 7 y PFS rates were $80 \%$ and $32 \%$, respectively. Median OS was NR in first line (95\% CI: 80, NE) or R/R pts (63, NE), with estimated 7 y OS rates of $75 \%$ and $52 \%$, respectively. Median OS for pts with PD within 1, 3, and $5 \mathrm{y}$ of treatment initiation was 13 (95\% CI: 1,

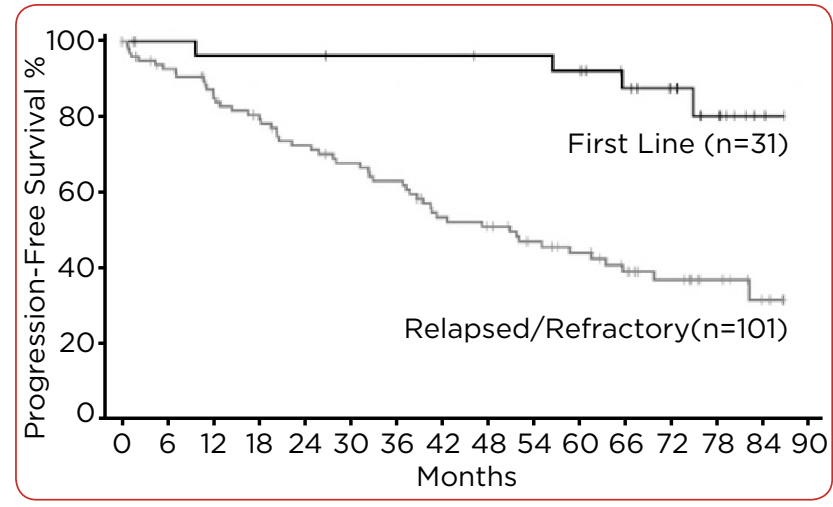

Figure 1. PFS for All-Treated First Line and Relapsed/Refractory Patients with CLL 
25), 28 (17, 54), and 57 mo (28, NE), respectively. In $\mathrm{R} / \mathrm{R}$ pts, median PFS was 26 mo (95\% CI: 18, $37)$ in 34 pts with del( $17 \mathrm{p}), 51 \mathrm{mo}(31,62)$ in $28 \mathrm{pts}$ with del(11q), 82 mo (7, NE) in 5 pts with trisomy 12 , and NR $(63, \mathrm{NE})$ in 13 pts with del(13q). Median PFS was NR (95\% CI: 40, NE) in $16 \mathrm{R} / \mathrm{R}$ pts with no abovementioned cytogenetic abnormalities. Among R/R pts, median PFS trended longer for 27 pts with 1-2 prior lines of therapy (66 [95\% CI: 37, NE]) versus 14 pts with $3(59[22, \mathrm{NE}])$ or 60 pts with $\geq 4$ prior lines of therapy $(39[26,51])$. The primary reason for treatment discontinuation in first line pts was AEs (23\%), whereas in R/R CLL it was PD (35\%). Most common AEs ( $\geq 2$ pts) leading to discontinuation were diarrhea, subdural hematoma, and sepsis ( $\mathrm{n}=2$ each). Grade $\geq 3$ AEs were reported in $74 \%$ of first line and $89 \%$ of $R / R$ pts, and serious AEs were reported in $61 \%$ of first line and $76 \%$ of R/R pts. Hypertension (first line, $32 \% ; \mathrm{R} / \mathrm{R}, 26 \%$ ) and pneumonia (first line, $10 \%$; $\mathrm{R} / \mathrm{R}, 27 \%$ ) were among the most common grade $\geq 3$

The Advanced Practitioner Perspective: Sandra E. Kurtin, PhD, ANP-C, AOCN ${ }^{\circledR}$

The ability to continue treatment, in this case, single-agent ibrutinib, with effective disease control and acceptable safety for up to 7 years of follow-up in both treatment-naive (TN) and relapsed or refractory $(R / R)$ chronic lymphocytic leukemia (CLL) or small lymphocytic lymphoma (SLL), even in patients with high-risk features, was the focus of this abstract. At 67 months' median follow-up, the duration of response (DOR) in patients who were newly diagnosed $(n=31)$ had not been reached. Among the 101 patients with $R / R$ disease, the average DOR was 4.7 years.

The 7-year progression-free survival (PFS) in this group of patients was estimated to be $80 \%$ for TN patients and 32\% for patients with R/R disease, again showing that single-agent ibrutinib could be very effective in these patients, despite the presence of unfavorable prognostic features. Overall survival was estimated to be $75 \%$ for TN patients and $52 \%$ in the R/R group, including those who were heavily pretreated prior to starting ibrutinib. In addition, R/R patients who received single-agent ibrutinib as an earlier line of treatment tended to have improvement in PFS. The primary reason for treat- treatment-emergent AEs. A similar proportion of first line (10\%) and R/R pts (9\%) experienced major hemorrhage. Grade $\geq 3$ atrial fibrillation (first line, $6 \% ; \mathrm{R} / \mathrm{R}, 10 \%$ ) and infection (first line, $23 \%$; $\mathrm{R} / \mathrm{R}, 55 \%$ ) were more common in $\mathrm{R} / \mathrm{R}$ pts. In all pts, grade $\geq 3$ treatment-emergent AEs were highest during the first year of treatment and subsequently declined.

Conclusions: With up to 7 y of follow-up, sustained activity of single-agent ibr was observed with first line ibr and for R/R pts with CLL/SLL, including those with high risk genomic factors. Responses were durable, with stable or improved CR rates, and sustained PFS and OS rates (estimated $7 \mathrm{y}$ rates: $80 \%$ and $75 \%$ for first line pts and $32 \%$ and $52 \%$ in the highly pretreated $\mathrm{R} / \mathrm{R}$ population, respectively). In $\mathrm{R} / \mathrm{R}$ CLL, ibr administration in earlier lines of therapy resulted in improved PFS outcomes, with longer PFS after 1-2 versus $\geq 3$ prior lines of therapy. Ibr was acceptably tolerated, and most grade $\geq 3$ AEs declined over time.

ment discontinuation in first-line patients was adverse events (AEs) (23\%), whereas in R/R CLL it was progressive disease (35\%). Grade 3 or greater treatment emergent adverse events (TEAEs) were reported in $61 \%$ of $\mathrm{TN}$ and $76 \%$ of $R / R$ patients. The most commonly reported grade $\geq 3$ TEAEs were hypertension and pneumonia. Major hemorrhage (10\% TN; 9\% R/R), grade $\geq 3$ atrial fibrillation ( $6 \% \mathrm{TN} ; 10 \% \mathrm{R} / \mathrm{R}$ ), and infection ( $23 \% \mathrm{TN}$; $55 \% \mathrm{R} / \mathrm{R}$ ) were also reported. These were most common in the first year of therapy and generally improved with continued treatment.

\section{The Role of the Advanced Practitioner}

Symptom management is a primary component of the AP role. This study illustrates the importance of anticipating and mitigating TEAEs to maintain treatment that has shown long-term benefit. As more therapies are coming to market, there is the temptation to change therapy prematurely instead of aggressively managing toxicity using the guidelines for dose modifications or treatment interruptions outlined in the clinical trials. In a patient with an incurable cancer such as CLL/SLL, maximizing therapies that provide long-term efficacy with acceptable safety may preserve future treatment options. 
Abstract 214

Dual Inhibition of Bruton's Tyrosine Kinase and BCL2: A Promising Therapeutic Strategy for Myeloid and Lymphoid Leukemias

Christopher A. Eide, Stephen E. Kurtz, PhD, Andy Kaempf, Cristina E. Tognon, PhD, Motomi Mori, PhD, Max J. Gordon, $M D$, Jeffrey $W$. Tyner, PhD, Alexey V Danilov, MD, PhD, and Brian J. Druker, MD

Visit http://www.bloodjournal.org/content/132/ Suppl_1/214 for a complete list of contributor affiliations and full graphics.

Background: The identification of effective therapies based on targeted interventions for human cancers faces the challenges of genetic and epigenetic heterogeneity underlying the disease. Large-scale sequencing efforts have uncovered a spectrum of mutations in many hematologic malignancies, suggesting that combinations of agents will be required to treat these diseases effectively. Combinatorial approaches will also be critical for combating the emergence of genetically heterogeneous subclones, rescue signals in the microenvironment, and tumor-intrinsic feedback pathways that all contribute to disease relapse.

Methods: We used a functional ex vivo screening assay to identify small-molecule target- ed inhibitors and inhibitor combinations demonstrating selective efficacy across broad categories of leukemia. Primary mononuclear cells isolated from leukemia patients $(n=588)$ were plated in the presence of a panel of graded concentrations of over 120 single-agent inhibitors or combinations spanning multiple drug classes. Leukemia specimens from 5 diagnosis subgroups included acute myeloid leukemia (AML; $n=293$ ), acute lymphoblastic leukemia (ALL; $\mathrm{n}=83$ ), chronic lymphocytic leukemia (CLL; $n=140$ ), chronic myeloid leukemia (CML; n=26) and myeloproliferative neoplasms/myelodysplastic syndrome (MPN or MDS/MPN; $\mathrm{n}=46$ ) patients. The combinations were designed as drug pairs that target non-overlapping biological pathways and comprise drugs from different classes, preferably with Food and Drug Administration approval. $\mathrm{IC}_{50}$ and AUC values were derived from probit-based regression for each response curve. Mutational status for FLT3ITD and NPM1 were compiled from clinical labs or by capillary electrophoresis using a QiaXcel instrument. Disease status was obtained from clinical chart review. Single and combination drug treatment $\mathrm{IC}_{50}$ and AUC values were compared within groups by Friedman test, across groups by Kruskal-Wallis test, and with continuous variables by Spearman rank correlation.

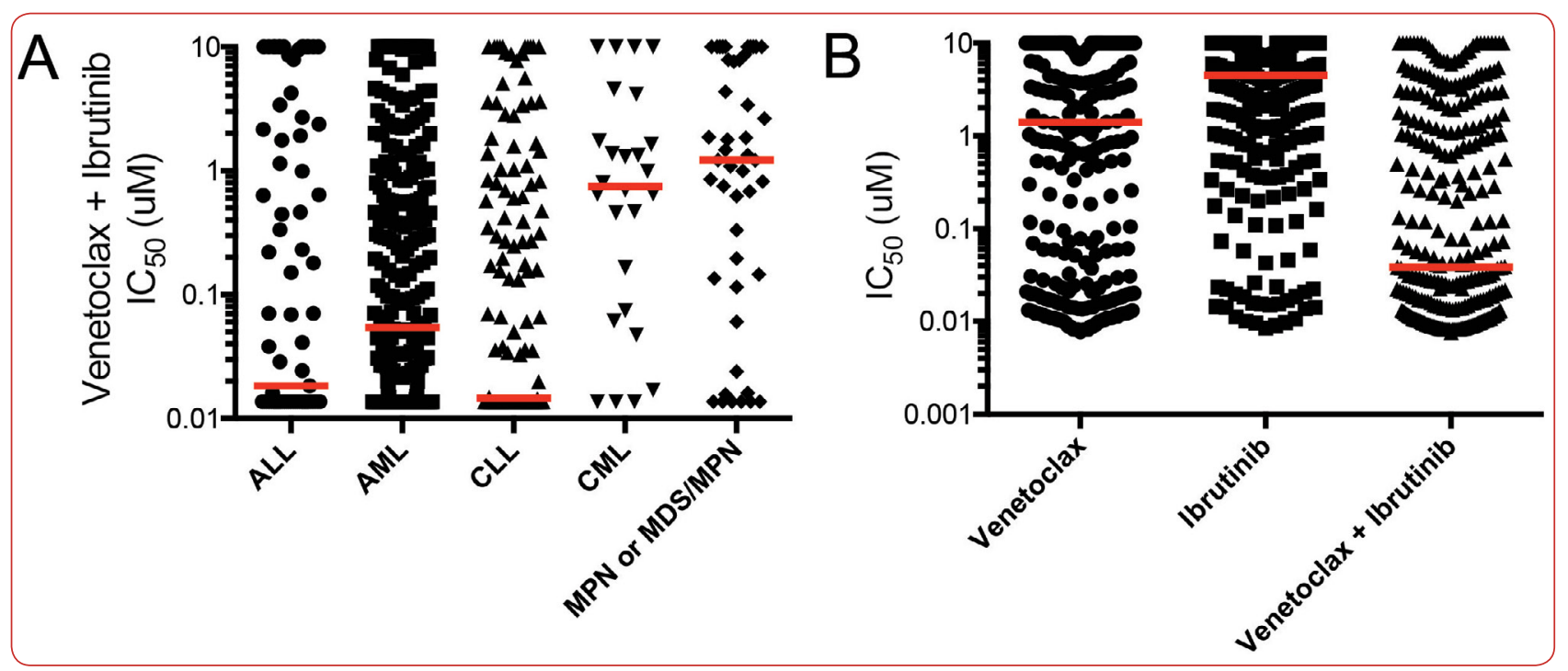

Figure 1. (A) Plots by IC50 sensitivities on all patients $(n=588)$ displayed by diagnosis subgroup. Red horizontal bars indicate the median values. Comparisons were performed using Kruskal-Wallis test adjusted for multiple comparisons. (B) Plots by IC50 sensitivities on AML patients $(n=293)$. Red horizontal bars indicate the median values. Comparisons were performed using Friedman test adjusted for multiple comparisons. 
Results: Among 588 unique leukemia patients evaluated, we observed that the combination of venetoclax (a BCL2 inhibitor) with ibrutinib (a BTK inhibitor) showed enhanced benefit above that for either single agent in both myeloid and lymphoid malignancies, including AML, ALL, and CLL. Expanded analyses of combination sensitivity in AML patients showed no significant associations with age, gender, or ELN prognostic risk. Comparison of AML samples by disease treatment status indicated a slightly greater sensitivity in relapsed/refractory $(n=42)$ versus newly diagnosed ( $\mathrm{n}=115)$ AML samples by both $\mathrm{AUC}$ and $\mathrm{IC}_{50}(\mathrm{p}=0.028$ and 0.026 , respectively). Among AML samples, FLT3-ITD and NPM1 mutation status was available for 204 and 188 patients, respectively. Venetoclax + ibrutinib was significantly more effective in patient samples harboring FLT3-ITD or NPM1 mutations by both AUC $(p=0.0002$ and $p=0.0032$, respec-

The Advanced Practitioner Perspective:

Sandra E. Kurtin, PhD, ANP-C, AOCN ${ }^{\circledR}$

Among the abstracts showing some of the progress in basic science is Abstract 214, a study that's very interesting to me. This trial looked at 588 patients with a variety of leukemias and other myeloid malignancies: AML $(n=293), C M L(n=26), A L L(n=83), M D S$ or MDS/MPN $(n=46)$. The primary focus was evaluating the impact of therapeutic strategies using agents with non-competing pathways, specifically the BTK inhibitor ibrutinib, and the B-cell lymphoma 2 (BCL2) inhibitor venetoclax. Using patient samples and testing for $\mathrm{IC}_{50}$ and AUC values, combining ibrutinib, a BTK inhibitor, and venetoclax, a BCL2 inhibitor, in patients that harbored FLT3 and NPM1 showed the two-drug combination to be significantly more effective (AUC; $p=.0002$ and $p=.0032$, and $\mathrm{IC}_{50} ; p=.0002$ and $p=.021$, respectively) than either agent alone.

Importantly, among 112 AML patients in this study, expression of BCL2 and BTK was tively) and $\mathrm{IC}_{50}(\mathrm{p}=0.0002$ and $\mathrm{p}=0.021$, respectively). Furthermore, among 112 AML samples with RNAseq data available, sensitivity to the combination (AUC) was significantly correlated with gene expression for BCL2 and BTK (Spearman $\mathrm{r}$ : -0.48 and -0.21 , respectively). Evaluation of the combination using an expanded $7 \times 7$ concentration matrix on AML cell lines revealed synergy for the majority of drug-pair concentrations by the Bliss independence model. Analysis to align additional clinical and genetic features for leukemia patient samples with drug sensitivities is in progress and results will be presented at the meeting.

Conclusion: Both myeloid and lymphoid-derived primary leukemia cells show sensitivity to combined inhibition of BCL2 and BTK with the combination of venetoclax and ibrutinib, suggesting this drug pair may have broad therapeutic indications.

statistically significantly correlated with improved response to the combination of ibrutinib and venetoclax in these patients (Spearman $r:-0.48$ and -0.21 , respectively). Although this is not yet an approved therapy, this study provides an important proof of concept in that these agents can be used across myeloid and lymphoid malignancies and can be combined to increase benefit and therapeutic outcome for selected patients with disease that harbors these attributes.

\section{Awareness of Clonal Evolution}

This abstract emphasizes the heterogeneity of each disease. It is important to note that individual patients may not exhibit these molecular targets at the time of diagnosis, but may acquire subclones that become dominant over time through the process of clonal evolution. For advanced practitioners, it is critical to test for known and emerging actionable targets and molecular attributes, both at baseline and at any point of progression. 


\section{Abstract 695}

First Prospective Data on Impact of Minimal Residual Disease on Long-Term Clinical Outcomes After Venetoclax Plus Rituximab Versus Bendamustine Plus Rituximab: Phase III MURANO Study

Arnon P. Kater, MD, Peter Hillmen, MB ChB, PhD, FRCP, FRCPath, Barbara Eichhorst, Anton W. Langerak, PhD, Thomas J. Kipps, MD, PhD, Carolyn Owen, MD, FRCPC, Michelle Boyer, Kathryn Humphrey, Elizabeth A. Punnoose, Jue Wang, Brenda Chyla, Maria E. Verdugo, Jenny Wu, Yanwen Jiang, Mehrdad Mobasher, MD, MPH, and John F. Seymour, MBBS, PhD

Visit http://www.bloodjournal.org/content/132/ Suppl_1/695 for a complete list of contributor affiliations and full graphics.

Introduction: Although CLL minimal residual disease (MRD) status is used in contemporary clinical trials aimed at maximizing response or determining treatment duration, its role as a predictive factor for PFS has only been established following chemoimmunotherapy. In contrast, whether MRD is a valuable tool for treatment choice in the era of novel targeted agents for CLL is unknown. Unlike kinase inhibitors, the BCL2 inhibitor venetoclax does result in undetectable MRD (uMRD). MURANO demonstrated significant PFS benefit for venetoclax + rituximab (VenR) given for a fixed duration vs bendamustine + rituximab (BR) in relapsed/refractory $(\mathrm{R} / \mathrm{R}) \mathrm{CLL}$. Here we present analysis of peripheral blood (PB) MRD kinetics in relation to PFS in MURANO with long follow up, when all pts have completed therapy.

Methods: Pts were randomized to VenR (Ven $400 \mathrm{mg} / \mathrm{d}$ for $2 \mathrm{yrs}+\mathrm{R}$ for first $6 \mathrm{mo})$ or BR (6 mo). Response was assessed clinically using complete blood count and physical exam at follow-up visits. PB MRD was analysed centrally by ASO-PCR and/or flow cytometry at Cycle 4, end of combination therapy (EOCT; mo 9) and every 3 mo thereafter until $3 \mathrm{yrs}$, then every 6 mo. As strong concordance between PB and bone marrow (BM) MRD with VenR was previously shown (Hillmen et al. ASCO 2018), we focus on PB MRD. Pts were categorized into UMRD ( $<1$ CLL cell per 10,000 leukocytes $\left.\left[<10^{-4}\right]\right)$, intermediate (int)-MRD+ $\left(\geq 10^{-4}-<10^{-2}\right)$, and high-MRD+ $\left(\geq 10^{-2}\right)$ status. Results are reported for the intention-to-treat (ITT) population.

Results: As of May 8 2018, median follow-up was 36.0 mo. VenR pts achieved high PB uMRD rates at $\mathrm{EOCT}$ ( $62 \%$ vs $13 \%$ for $\mathrm{BR}$ ). The same pattern was observed through mo 24 , when Ven single-agent treatment was scheduled to cease: $48 \%$ uMRD in VenR vs $2 \%$ in BR, and $16 \%$ int$\mathrm{MRD}+$ in VenR vs 7\% in BR (Table 1). By then, $18 \%$ pts in the VenR arm had progressed/died/ withdrew from study vs $66 \%$ in BR. Consistently high uMRD rates were observed in all VenR subgroups, including pts with high-risk cytogenetics and molecular factors: $\operatorname{del}(17 \mathrm{p})$ and/ or TP53 mutated: $57 \%$ at EOCT and $36 \%$ at mo 24; IGHV unmutated: $61 \%$ at EOCT and $51 \%$ at mo 24.

Landmark analysis of PFS by MRD status in PB at the EOCT response visit showed that, regardless of treatment arm, uMRD status was associated with longer PFS. Within MRD+ pts, the int-MRD+ group had improved PFS over high-MRD+ pts (Fig 1). Due to the very low rate of $\mathrm{uMRD}$ in the $\mathrm{BR}$ arm, all further analyses were performed in VenR pts; PFS curves overlapped for pts with uMRD, regardless of whether in CR or PR by investigator assessment, while inferior PFS for MRD+ PR/nPR

\begin{tabular}{|c|c|c|c|c|}
\hline \multirow[b]{2}{*}{$\%$ of pts } & \multicolumn{2}{|c|}{ VenR $(n=194)$} & \multicolumn{2}{|c|}{ BR $(n=195)$} \\
\hline & Mo 9 (EOCT) & Mo 24 & Mo 9 (EOCT) & Mo 24 \\
\hline UMRD & 62 & 48 & 13 & 2 \\
\hline Int-MRD+ & 19 & 16 & 23 & 7 \\
\hline High-MRD+ & 5 & 11 & 29 & 18 \\
\hline Missing & 7 & 7 & 15 & 7 \\
\hline PD/death/withdrew & 7 & 18 & 20 & 66 \\
\hline
\end{tabular}


pts becomes manifest from mo 18 after EOCT (Fig 2). MRD+ pts in CR (all int-MRD+) did as well as UMRD, but numbers are very small.

130 pts in VenR arm completed 2 yrs Ven treatment without PD; among them: 83 (64\%) were uMRD, 23 (18\%) were int-MRD+, 14 (10\%) were high-MRD+ and 10 (8\%) had missing data at mo 24. With 9.9 mo median follow-up from Ven completion, amongst uMRD pts at mo 24 , the majority remained uMRD (57/83, 69\%) and 26/83 (31\%) converted to confirmed $\mathrm{MRD}+$ (2 serial assay positive). Conversions were mainly to int-MRD+ (21/26, 81\%), all of whom remain PFS event-free; $19 \%(5 / 26)$ converted to high-MRD+, 2 had PD per iwCLL criteria (Fig 3). Of pts converting from uMRD to MRD+ after cessation of Ven at mo 24, $40 \%(10 / 25)$ carried del(17p) and/or TP53mutation, vs $23 \%(12 / 53)$ among those who did not convert. Of int-MRD+ pts at mo 24, 9/23 (39\%) became high-MRD+ (2 had PD); there was $1 \mathrm{PD}$ among the remaining 14 . PD was seen in $11 / 14$ (79\%) of pts who were high-MRD+ at mo 24 .
Conclusion: The higher PB UMRD rate observed at EOCT in the VenR arm vs BR arm was maintained at completion of Ven treatment, including in high-risk subgroups, consistent with maintained PFS benefit seen with longer follow up. VenR pts who achieved uMRD or int-MRD+ had durable PFS. MRD status powerfully identified distinct outcomes for pts with PR/nPR; longer follow-up is needed to determine the impact of MRD status among CR/CRi. In the first yr post-completion of fixed-duration VenR treatment, majority of uMRD pts remained $\mathrm{uMRD}$; reemergence of $\mathrm{MRD}+$, mainly at int levels, is seen in a minority of pts, and has shown a low rate of progression to clinical $\mathrm{PD}$ to date. These data are the first to demonstrate the value of $\mathrm{PB}$ MRD as a predictive marker of clinical outcome for the fixed-duration chemotherapy-free regimen, VenR. Our data corroborate that uMRD and int-MRD+ rate is a meaningful endpoint and desirable goal of CLL therapies, including targeted agents.

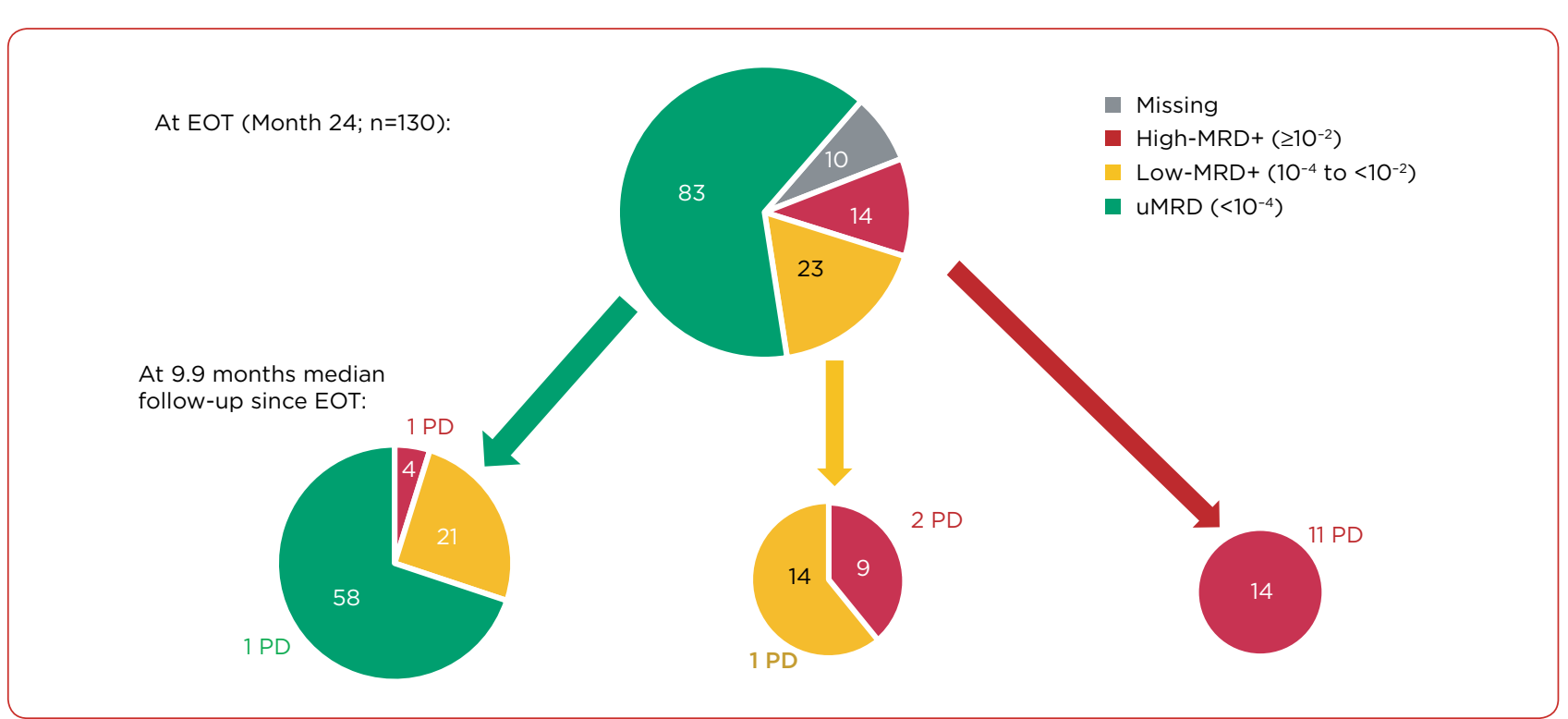

Figure 3. MRD changes in VenR arm patients who were progression-free at mo $24(n=130)$

The Advanced Practitioner Perspective: Sandra E. Kurtin, PhD, ANP-C, AOCN ${ }^{\circledR}$ Abstract 695 provided an update to the MURANO trial, a prospective evaluation of patients with CLL and SLL receiving either bendamustine and rituximab for 6 months (standard of care) or venetoclax and rituximab (comparison arm) for a total of 24 months. Data previously reported for the MURANO trial favored the venetoclax and rituximab arm (Seymour et al., 2017). Importantly, this study was looking at the use of peripheral blood to detect minimal 
COMMENTARY

KURTIN

residual disease. In patients receiving venetoclay and rituximab, 62\% achieved undetectable minimal residual disease (UMRD) status at the end of treatment, vs. $13 \%$ of the patients receiveing bendamustine and rituximab.

Among the 130 patients on the venetoclay and rituximab arm, at 2 years of followup, there was no progression of disease, and $69 \%$ of these patients sustained that response at 3 years of follow-up. In patients receiving the combination of venetoclax and rituximab, UMRD rates were observed at the end of cancen treatment across groups harboring del(17p) and/or TP53 mutated (57\%) or uIGHV (61\%). Among patients who relapsed, the majority of those harbored del(17P) or the TP53 mutation.

This abstract further demonstrates the inportance of monitoring patients for undetect- able minimal residual disease. It may also have implications for the need for extended therapy in patients with very-high-risk features. The ability to monitor UMRD using peripheral blood adds a significant advantage for patients and ADs. We will need to wait for regulatory decision on reimbursement and ongoing evaluatimon of UMRD in clinical trials to gauge the full impact of this study.

Reference

Seymour, J. F., Ma, S., Brander, D. M., Choi, M. Y., Barrientos, J., Davids, M. S.,... Roberts, A. W. (2017). Venetoclax plus rituximab in relapsed or refractory chronic lymphocytic leukaemia: A phase ib study. Lancet Oncology, 18(2), 230-240. https://doi.org/10.1016/S14702045(17)30012-8

J Adv Pact Oncol

32

AdvancedPractitioner.com 